Cahiers d'études africaines

$179-180 \mid 2005$

Esclavage moderne ou modernité de l'esclavage?

\title{
Paroles d'esclaves au Nord-Cameroun
}

Issa Sa ïbou

\section{OpenEdition}

Journals

Édition électronique

URL : http://journals.openedition.org/etudesafricaines/15030

DOI : 10.4000/etudesafricaines. 15030

ISSN : $1777-5353$

Éditeur

Éditions de l'EHESS

Édition imprimée

Date de publication : 19 décembre 2005

Pagination : 853-877

ISBN : 978-2-7132-2049-4

ISSN : 0008-0055

Référence électronique

Issa Sa ïbou, « Paroles d'esclaves au Nord-Cameroun », Cahiers d'études africaines [En ligne], 179-180 | 2005, mis en ligne le 01 janvier 2007, consulté le 16 juin 2020. URL : http://journals.openedition.org/ etudesafricaines/15030; DOI : https://doi.org/10.4000/etudesafricaines.15030

(C) Cahiers d'Études africaines 
chercher : repérer : avancer

Cet article est disponible en ligne à l'adresse :

http://www.cairn.info/article.php?ID_REVUE=CEA\&ID_NUMPUBLIE=CEA 179\&ID_ARTICLE=CEA 1790853

Paroles d'esclaves au Nord-Cameroun

par Issa Sa ÏBOU

\section{Editions de l'EHESS | Cahiers d'études africaines}

2005/3-4 - 179

ISSN 0008-0055 | ISBN 2713220491| pages 853 à 877

Pour citer cet article :

_ ïbou I., Paroles d'esclaves au Nord-Cameroun, Cahiers d'études africaines 2005/ 3-4, 179, p. 853-877.

Distribution électronique Cairn pour Editions del'EHESS.

(C) Editions de l'EHESS. Tous droits réservés pour tous pays.

La reproduction ou représentation de cet article, notamment par photocopie, n'est autorisée que dans les limites des conditions générales d'utilisation du site ou, le cas échéant, des conditions générales de la licence souscrite par votre établissement. Toute autre reproduction ou représentation, en tout ou partie, sous quelque forme et de quelque manière que ce soit, est interdite sauf accord préalable et écrit de l'éditeur, en dehors des cas prévus par la législation en vigueur en France. Il est précisé que son stockage dans une base de données est également interdit. 


\section{Paroles d'esclaves au Nord-Cameroun}

Dans le processus de collecte des données en vue de la rédaction de ce texte, il nous a été donné de réaliser combien les faits divers déterminent le cours des relations humaines dans la partie septentrionale du Cameroun. Lors de discussions à bâtons rompus au cours desquelles, sur les sujets délicats, l'informateur est plus disposé à causer avec le chercheur qu'à répondre à un questionnaire en prenant des notes, on apprend énormément sur les conflits pérennes entre gens de pouvoir, sur les méandres de l'accumulation de tel nanti ou sur les causes de l'émigration de telle famille. À longueur de journée, l'ombre des grands arbres qui protège les hommes de la canicule de mars à mai favorise la relation d'histoires de vie. C'est là que nous avons débuté les recherches préliminaires pour l'enquête de terrain qui a produit ce texte. Parmi la dizaine de personnes présentes figurait un vieillard qui nous avait été recommandé. Il ne tarissait pas de petites histoires croustillantes personnelles ou concernant des connaissances, au sujet notamment des relations entre maîtres et esclaves avant l'indépendance. Mais notre tentative d'engager une interview formelle eut un effet amnésique immédiat. Plusieurs autres tentatives aboutirent au même résultat. C'est seulement en transitant par le canal d'un groupe d'action contre les cultures discriminatoires, en usant d'affinités et d'accointances amicales, professionnelles, familiales, estudiantines et autres parmi des descendants de la classe servile, que nous avons pu avoir accès aux témoignages de ceux qui, d'une façon ou d'une autre, ont vécu la servitude dans le Cameroun d'après les indépendances.

Quoique provenant généralement de familles dont les derniers membres sont affranchis depuis au moins une vingtaine d'années, nos mentors dans cette collecte de données ont, par intérêt pour le sujet, mis en évidence l'actualité du phénomène servile. C'est à leur demande expresse et avec l'assentiment des interviewés que nous employons de préférence les termes d'esclavage et d'esclave plutôt que les euphémismes de servitude ou de domesticité introduits par le colonisateur pour ne pas exprimer la réalité vécue. Il a été parfois révoltant de recueillir le témoignage de personnes 
subissant le présent sur la base de codes religieux et sociaux mal assimilés et hors contexte.

Nous tenterons de répondre ici à une partie des préoccupations de Paul E. Lovejoy (2000 : 287-289) en ce qui concerne le legs de l'esclavage: «Even when slaves were not formally emancipated or locally not recognized as free, the social and political setting had changed irrevocably [...] the question is not whether or not there was a dramatic break with the past, but the ways in which people were able to shape the new order, preserving the old or rising above it. Much colonial and post-colonial scholarship has to be re-examined with the problem of abolition in mind. » Ce qui, en revanche, relativise la distinction entre slaveholder et slaveowner établie par Kevin Bales (2004 : 5) afin de différencier l'esclavage contemporain de l'esclavage ancien sur la base de la légalité du droit de propriété, dès lors que « Today slavery is illegal everywhere, and there is no more legal ownership of human beings. »

Nous verrons qu'au Nord-Cameroun ce n'est pas l'État qui applique la loi contre l'esclavage, mais seulement les esclaves avisés qui se l'appliquent à eux-mêmes. Avant cela, nous replacerons les processus d'asservissement dans leur contexte et nous analyserons la reproduction de l'esclavage royal. Nous donnerons ensuite la parole aux victimes de l'exploitation au fur et à mesure de l'analyse.

\section{Des processus d'asservissement au Nord-Cameroun}

La traite humaine, dans l'histoire des abords sud du lac Tchad où se situe le Nord-Cameroun, prend prétexte dans le prosélytisme islamique des grandes hégémonies politiques du bassin tchadien. En effet, depuis l'introduction de l'islam à la cour du Kanem à la fin du XI ${ }^{\mathrm{e}}$ siècle, les conquêtes territoriales et les razzias esclavagistes que perpétraient déjà les royaumes du bassin tchadien ont été anoblies sous couvert de desseins religieux. Ainsi, quand au $\mathrm{XVI}^{\mathrm{e}}$ siècle le Maï du Bornou, Idris Aloma, entreprit de réduire les Sao pour des raisons essentiellement défensives, son historiographe (Ibn Fartua 1970 : $10,13)$ n'y vit que la manifestation de sa foi sur le chemin d'Allah : «How then about the exemplary punishment he meted out to the tribe of So, in accord with God's command to fight unbelievers, who are close to Muslims and vexatious to them [...]. His actions covered most of that which is ordained in the Kurà'an and Sunna concerning "Holy War" in the path of God, seeking the noble presence of God, and His great reward. »

Du Bornou au Baguirmi en passant par le royaume du Wandala, les cours royales qui s'étaient investies de la mission de répandre l'islam répandirent davantage le sang des «infidèles » que la foi elle-même; le terme « infidèle » signifiant plus celui qui est « réductible en esclavage » que celui qui résiste à l'appel à la conversion volontaire, gage de vie sauve et du maintien de la liberté individuelle. Il est évident que l'islam fut plus diffusé 
par l'action isolée des marabouts et l'adhésion volontaire des populations à la religion des détenteurs du pouvoir que par la force du glaive. Faites au nom de l'islam mais pour le compte des gouvernants, les campagnes militaires procédaient manifestement du banditisme d'État, un mode de production économique en deux volets : razzias du bétail animal et razzias du bétail humain.

En effet, bétail animal et bétail humain constituaient les deux principaux produits d'échanges dans les marchés dont celui de Kukawa ${ }^{1}$ était le mieux achalandé en variétés de marchandises humaines. Plus que les conquêtes territoriales et la protection des frontières de l'État dans un contexte d'inimitié généralisée, les razzias formaient la raison d'être essentielle de l'armée ; elles renflouaient les caisses des dignitaires dont l'engagement dans telle ou telle campagne coïncidait souvent avec l'amenuisement de leurs avoirs. Les razzias permettaient aussi de rétribuer les soldats en même temps qu'elles étaient l'occasion pour des cavaliers isolés, tels les Arabes Choa, de se joindre à l'armée officielle et de participer à la collecte de la marchandise en pays mousgoum ou massa ${ }^{2}$. Le fait que des esclaves, quoique dignitaires serviles, aient eux-mêmes possédé des esclaves montre que l'islam ne fut nullement le fondement de ces razzias. L'extermination des razzieurs se faisait avec une telle violence que le nombre des morts qu'ils laissaient sur le terrain et celui des captifs qu'ils faisaient n'étaient parfois pas très éloignés l'un de l'autre. Aux milliers de captifs saisis, il faut ajouter autant de décapités, de personnes cueillies par des flèches en haut des arbres dont le feuillage servait de refuge et d'autres encore bousculés et piétinés par les chevaux, ou encore des vieillards et des enfants morts de fatigue ou de faim en cours de route. La description des razzias commises sur les peuples dits païens du Nord-Cameroun, en particulier sur les Mousgoum de la vallée du Logone, traduit l'horreur de la besogne qui pourtant fit la fierté des armées précoloniales. Ce que la littérature appelle « campagnes » ou « expéditions », pour désigner les opérations militaires du Bornou, du Baguirmi ou du Wandala, est semblable à la « pacification », terme usité pour nommer les opérations de police menées par le colonisateur français contre les Kirdi ${ }^{3}$ réfractaires au système colonial. Au milieu du XIX ${ }^{\mathrm{e}}$ siècle, Barth (1965 : 410) résume bien le sentiment qu'inspirent les razzias à l'observateur :

«I lamented the misery of accompanying such an expedition; for nothing can be more disheartening to the feelings of a traveller who is desirous of knowledge than

1. Kukawa ou Kouka était la capitale du Bornou sous le règne de la dynastie des Shehou. La ville est située sur la rive occidentale du lac Tchad, au nord d'Angornou. À la fin du XIX ${ }^{\mathrm{e}}$ siècle, le conquérant Rabah déplaça la capitale à Dikoa.

2. Les Massa et les Mousgoum habitent la vallée méridionale du fleuve Logone. On les retrouve de part et d'autre de cette frontière naturelle entre le Cameroun et le Tchad. Au Cameroun, ils se concentrent dans le département du Mayo Danay, dans la province de l'Extrême-Nord. Ils sont généralement grands de taille et robustes.

3. Expression désignant les peuples non islamisés du Nord-Cameroun. 
to visit these beautiful countries under such circumstances, when the original inhabitants are either exterminated, or obliged to seek their safety in flight; all traces of their cheerful life are destroyed, and the abodes of human happiness converted into desolation. The stranger who intrude upon the natives in this hostile manner [...] is at least justified in speaking more emphatically of the endless misery into which the finest and most populous regions of this continent are plunged by these slavehunting expeditions of their merciless Mohammedan neighbours. »

Peut-être comprend-on mieux ici les fondements de la diversité des moyens de se soustraire aux esclavagistes, développés par les peuples matés. Car, hormis le retrait dans des sites insalubres ou escarpés, la tradition orale admet l'enlaidissement des femmes comme moyen de se rendre hideuses, « inconcubinables », dans la mesure où les razzias étaient aussi l'occasion de renouveler les harems. Sachant combien Mousgoum et Massa donnent de l'importance au corps, à son apparence, l'on pressent qu'au-delà de leur fonction culturelle, les labrets ${ }^{4}$ que portaient les femmes semblent aussi avoir eu pour fonction d'éviter l'esclavage en servant de repoussoir. D'ailleurs, le Major Denham corrobore bien ce fait lorsqu'il décrit les femmes mousgoum assignées aux travaux champêtres aux abords de Kukawa:

«Les marchands de Tripoli et du Fezzan ne les achètent jamais à cause de leur laideur, parce qu'ils ne pourraient pas les vendre. Elles ont de gros vilains traits, et de plus sont très défigurées par le clou d'argent qu'elles portent à la lèvre inférieure ; d'ailleurs on leur ôte deux dents inférieures pour faire place au morceau de métal qui pénètre tout à fait dans leur bouche; et, au bout d'un an ou deux, le poids du métal fait tomber entièrement la lèvre sur le menton, de sorte que leur figure est horrible» (Denham et al. $1826:$ 66).

En plus de leur valeur en tant qu'objet de vente dans les échanges extérieurs notamment, les esclaves furent le fer de lance de l'expansion territoriale des royaumes en même temps que le support de l'économie interne. Thierno Bah (1993 : 71 et 74-75) souligne parfaitement le rôle militaire des esclaves: ces «Peul [qui] mettent leur zèle de néophytes au service du prosélytisme religieux » avaient néanmoins incorporé les populations locales dans les armées dont elles composaient le gros des troupes, de telle sorte qu' «après les premières flambées du jihad, une alliance s'établit entre les Peul et certains peuples ». Cette coalition islamo-animiste fut à l'origine des ponctions humaines au détriment de peuples tels que les Laka, et qui produisaient, chaque année, entre 8000 et 10000 esclaves assignés dans des colonies agricoles, les dumde (sing. rumde) (Bah 1982). Barth, qui visita ces colonies dans l'actuelle province de l'Adamaoua, rapporte ceci (1965: 190-191) :

4. Le commandant Lenfant (1905: 145-146) décrit les labrets comme suit: «Dès le jeune âge, la fillette se perce les lèvres et passe dans chaque trou des rondins de bois gros comme des allumettes, puis, progressivement, augmente le diamètre de ces ornements, en sorte qu'une femme adulte peut orner sa bouche avec des rondelles de bois larges comme des assiettes à dessert. » 
«Slavery exists on an immense scale in this country, and there are many private individuals who have more than a thousand slaves. In this respect the governor of the whole presence is not the most powerful man, being outstripped by the governors of Chamba and Koncha for this reason, that Mohammed Lowel has all his slaves settled in rumde or slave-villages, where they cultivate grain for his use or profit, while the above mentioned officers, who obtain all their provision in corn from subjected pagan tribes, have their whole host of slaves constantly at their disposal ; and I have been assured that some of the head slaves of these men have as many as a thousand slaves each under their command, with whom they undertake occasional expeditions for their masters. »

Dédaignant l'agriculture comme bien des peuples nomades, porteurs du message islamique à répandre par l'épée — quoique habités pour la plupart d'une islamité superficielle — mais disposant de forces militaires insuffisantes, rancuniers à cause des souffrances endurées du temps de leur assujettissement aux chefs kirdi, les Peuls trouvèrent dans l'esclavage le moyen d'asseoir une hégémonie revancharde, idéologique et fondée sur l'exploitation. Outre l'exploitation politique et économique des esclaves, les dignitaires firent des asservis un objet de plaisir en s'entourant de concubines, et un objet de protection du harem et du sérail par l'emploi d'eunuques. L'émasculation de ces hommes dans des sociétés où la masculinité se mesure autant à la bravoure qu'à la virilité sexuelle de l'homme, fait de l'homme castré un déshumanisé qui côtoie les femmes... du regard. Tout comme l'horreur des razzias, celle de la castration apparaît terrifiante. À ce propos, Gustav Nachtigal (1980: 217) rapporte ce qui suit:

«Under the pretext of wanting to circumcise the boys, the barbers who perform the operation are accustomed with a quick grip to grasp the whole of their external genitals in the left hand, and with the right to amputate them with a sharp knife. Boiling butter is kept in readiness and poured on the fresh wound to staunch the bleeding of the unfortunate boys. Very many of them, of course, succumb to the horrible operation. »

Aussi majestueuses qu'aient pu être l'ascension et les faveurs obtenues par les esclaves de cour en général, il reste qu'ils ne furent jamais à l'abri d'un rappel brutal de leur condition servile. Dignitaire de confiance, oncle du souverain régnant, chef des armées, gouverneur de province ou laboureur, l'esclave restait un captif dont la promotion sociale ne s'opérait qu'à l'intérieur d'une hiérarchie servile. L'exemple des esclaves de cour du califat de Kano qu'analyse avec érudition Sean Stilwell (2000) s'apparente bien à la condition qui est aujourd'hui la leur dans le lamidat de Rey Bouba, État traditionnel au sein de l'État camerounais. Dans un cas comme dans l'autre, la puissance du souverain, le rayonnement de son règne et la pérennité de son aura dépendent, dans une large mesure, de la subordination des esclaves, en contrepartie de l'accès à des positions de pouvoir qui sont autant de sources d'accumulation. Réputés arrogants et brutaux, les esclaves royaux 
agissent au nom mais pas toujours pour le compte du Baaba (le père), dont la seule évocation du nom confère l'impunité. À Rey Bouba, les temps anciens sont toujours présents ${ }^{5}$.

\section{Rey Bouba ou les survivances du royal slavery}

De toutes les chefferies traditionnelles camerounaises, Rey Bouba est celle qui conserve l'image de l'unité de commandement traditionnel ayant le mieux résisté aux sirènes et aux changements induits par la modernité. Dans l'esprit comme dans les méthodes et les propos des notables et autres serviteurs du Baaba, Rey Bouba et l'État camerounais sont deux entités similaires, entretenant des relations d'égal à égal. C'est ainsi que les «étrangers » qui manifestent des signes d'impatience face aux dogaris (la milice du Baaba) s'entendent généralement répondre que si ce type de comportement critique est tolérable au Cameroun, il ne l'est pas à Rey Bouba. État dans l'État, Baabaland, État féodal, État-musée, Hibernocratie, lamidat des âges farouches, telles sont quelques-unes des expressions par lesquelles divers observateurs du système en vigueur à Rey Bouba désignent cette entité de $36000 \mathrm{~km}^{2}$ abritant quelque 55000 âmes.

Perçu comme la propriété de son maître, le lamidat de Rey Bouba apparaît comme une excroissance, une espèce de relique de l'État précolonial, une survivance d'une Afrique réfractaire aux mutations politiques induites par la modernité. Ici, plus qu'ailleurs, le vocable laamiido (ou lamido) par lequel on désigne le chef traditionnel en fulfulde (la langue peule), revêt tout son sens et son contenu. Littéralement «l'intronisé », le terme de laamiido provient du mot laamu, signifiant alternativement pouvoir, autorité ou règne selon le contexte dans lequel il est employé. Il revêt tout d'abord une connotation répressive, véhicule une idée de domination, valorise l'obéissance envers le chef et le recours à la force pour briser toute forme de résistance. C'est ainsi que parmi les lamidats peuls du Nord-Cameroun ${ }^{6}$, ceux de Rey Bouba, de Mindif ou de Tchéboa - réputés pour la main de fer qui y caractérise l'exercice du pouvoir — ont de tout temps été considérés comme les grands lamidats du septentrion camerounais. "Le pouvoir mange les hommes », aimait à rappeler le lamido Ahmadou Bouhari de Mindif (19551991) (Sali Babani 1998). Cette expression signifie exactement que les sujets sont taillables et corvéables à merci, que le chef peut disposer de

5. Le lamidat de Ray ou Rey Bouba fait partie du grand émirat de l'Adamawa qui dépendait du califat peul de Sokoto. Sous l'administration française, il porta indistinctement le nom de lamidat de Bouba Ndjidda ou Rey Bouba. Aujourd'hui, ce commandement traditionnel épouse les frontières du département du Mayo Rey dans la province du Nord.

6. Les lamidats du Nord-Cameroun sont classés en chefferies de $1^{\text {er }}, 2^{\mathrm{e}}$ et $3^{\mathrm{e}}$ degrés (correspondant respectivement aux grandes et anciennes chefferies, aux cantons et aux villages). Parmi les principaux lamidats, on peut citer Ngaoundéré, Garoua, Maroua, Rey Bouba, Mindif, Kousseri, Goulfei, Logone Birni, Bogo. 
leur temps et de leurs biens selon ses souhaits. Nous avons là l'essence même des abus et autres exactions. Avant l'imposition au XIX ${ }^{\mathrm{e}}$ siècle de l'hégémonie peule sur une partie importante du Nord-Cameroun, l'ardo peul ou chef de clan conduisait lui-même son troupeau aux pâturages et payait tribut aux chefs païens. À Ngaoundéré, par exemple, ce sont les Mboum qui inculquèrent à l'ardo le sens du pouvoir avec ses fastes et sa faculté de contrainte. Le lamido ne pouvant arrêter, emprisonner et punir ses congénères peuls, c'est aux esclaves qu'échut cette tâche et ils s'en acquittèrent avec application. Quoiqu'entouré aussi bien de dignitaires peuls que de dignitaires asservis, c'est essentiellement avec ces derniers que règne l'ardo devenu lamido au point de susciter l'amertume résignée des Peuls. Les centenaires dont nous avons eu l'opportunité de recueillir les témoignages s'accordent pour dire qu'ils redoutaient plus le dogari que le lamido, car « le dogari faisait le lamido».

À Rey Bouba, le lamido Bouba Djama'a (1902-1945) comprit, dès les premiers contacts avec le colonisateur allemand, qu'une collaboration zélée et opportuniste lui garantirait les mains libres dans son lamidat. Il donna aux Allemands ce qu'ils voulaient, exécuta les ordres avec empressement. Il fit de même avec les troupes franco-britanniques lors de la Première Guerre mondiale. Puis, lorsque l'administration française s'installa au Cameroun, au lendemain de la guerre, Bouba Djama'a et ses successeurs firent preuve d'une collaboration à nulle autre pareille. En contrepartie, les Français n'installèrent que tardivement une subdivision administrative dans le lamidat. L'État postcolonial entérina les relations privilégiées entre Rey et le pouvoir central à Yaoundé. Aussi fallut-il attendre les années 1980 pour que soit créée une sous-préfecture dans le lamidat, mais on se garda bien d'établir le chef-lieu à Rey Bouba ville, là où réside le lamido. C'est à Tcholliré que s'installent le sous-préfet de l'arrondissement puis le préfet du département du Mayo Rey. Manifestement, on a voulu éviter de faire cohabiter dans la même ville l'autorité de l'État et celle du Baaba. En outre, la contribution du lamido Abdoulaye Ahmadou à la prééminence du parti au pouvoir dans le Mayo Rey, lors des élections multipartites consécutives à l'ouverture démocratique $^{7}$ n'a fait que renforcer la marge de manœuvre dont dispose le Baaba dans la gestion de son fief ${ }^{8}$.

L'attitude des souverains de Rey Bouba à l'égard des pouvoirs extérieurs participe donc d'une économie de la soumission, d'une logique de don et de contre-don : en contrepartie de la soumission absolue de Baaba aux autorités de Yaoundé, le pouvoir central le laisse soumettre ses sujets à des règles

7. En 1992, le parti de l'opposition UNDP (Union nationale pour la démocratie et le progrès) était sorti vainqueur des élections. En 1997, la victoire est allée au RDPC (Rassemblement démocratique du peuple camerounais). La presse avait fait état des divers blocages auxquels l'opposition fut confrontée aussi bien lors des campagnes électorales que pendant le déroulement du vote.

8. Sur la dynamique des relations entre les chefs de Rey Bouba et le pouvoir central, voir Taguem FaH \& Lamblin (2003). 
administratives et fiscales héritées de ses ancêtres. De ce point de vue, Rey Bouba présente les caractéristiques d'un État disposant de sa propre armée (les dogaris) contrôlant toutes les issues, de ses prisons où des gens peuvent être enfermés durant des années, de sa fiscalité, etc. Voici ce qu'écrit Valentin Zinga dans l'édition du journal Le Messager du 21 juin 2003 :

«Témoignage largement répandu : il a droit de vie et de mort sur ses sujets. Et de ce point de vue, il vaut mieux ne pas résister à ses desiderata. Exemple : il lève arbitrairement des impôts sur les récoltes des paysans, et les cheptels des éleveurs, et sur les commerçants, soutient une élite locale, bien renseignée, étouffant sous son indignation. [...] En vérité, le lamido a subdivisé son territoire en quatre zones contrôlées chacune par un de ses obligés, les dougaris. La zone de Touboro, vers la frontière avec le Tchad; la zone de Baïmboum, à la frontière avec la République centrafricaine ; la zone de Madigrim ; et la zone de Tcholliré (NDLR : localité célèbre pour abriter une prison politique sous les régimes successifs de Ahmadou Ahidjo et Paul Biya). À chacun de ses dougaris, il fixe des objectifs à atteindre. Une sorte de cahier des charges, qui détermine leur rendement sur les prélèvements à effectuer en sa faveur. On peut donc imaginer combien ils doivent être opiniâtres, ces dougaris, pour satisfaire le lamido [...]. Ainsi va cette chefferie singulière, moqueuse de la République et des droits de l'homme. »

Les agents de l'État en service dans le Mayo Rey sont considérés par les dogaris comme des invités. De ce fait, ils doivent se comporter comme tels, c'est-à-dire éviter de contrecarrer les représentants du Baaba, car cela serait interprété comme une ingérence dans les affaires intérieures du lamidat. L'on comprend mieux alors l'attitude du préfet Aboubakari Malam devant le lamido de Rey Bouba. Dans un article intitulé «La République à genoux », le journal Mutations relate une scène qui s'est déroulée le 26 mai 2003 et qui a défrayé la chronique :

«On peut voir le représentant personnel du chef de l'État et de ses ministres, un administrateur civil principal, c'est-à-dire la crème du personnel de commandement, en tenue de commandement, tout en sueur, l'échine courbée, les mains jointes en signe de soumission, le derrière en l'air et les genoux au sol, devant un représentant, fier, souriant et dominateur de l'aristocratie féodale la plus primitive ! Et ce sous le regard appréciateur voire approbateur de son patron le Minatd [Ministre de l'Administration Territoriale et de la Décentralisation], chantre comme on sait de la "restauration de l'autorité de l'État". Cette image d'une République couchée a de quoi choquer. Ce genre d'anachronisme est voulu et entretenu [...]. Honte à la République $»^{9}$.

L'autorité du Baaba sur ses sujets et le contrôle qu'il exerce sur toute l'étendue de son lamidat ne manquent pas cependant de soulever un certain nombre de questions concernant la permanence de l'insécurité due aux coupeurs de routes. Le Mayo Rey, pourtant le territoire le mieux surveillé du Cameroun, est paradoxalement l'un de ceux qui sont littéralement pris en otage par les bandits. Du fait de la présence dans cette région du marché

9. Mutations, 4 juin 2003. 
frontalier de Mbaimboum à la frontière avec le Tchad et la Centrafrique, l'activité commerciale y est fructueuse, tout comme le sont les opérations des bandits de grand chemin. S'il est avéré que les dogaris ont souvent fait la preuve de leur efficacité dans la répression des embuscades, un doute plane sur la nature exacte de leur attitude vis-à-vis des malfaiteurs. Ce que résume ainsi Abdoul Kadiri (2002: 64) :

«D'autres points de vue indiquent que depuis le début des années 1990, zarguinas $^{10}$ et dogaris cheminent ensemble. Ceux que les dogaris affirment avoir neutralisés seraient alors des intrus, des bandits étrangers qui veulent agir pour leur propre compte à l'insu des dogaris. Car, la découverte le 16 février 2001 dans un véhicule que conduisaient les bandits s'exprimant exclusivement en arabe choa, d'un contrat de versement d'une valeur de dix millions de francs CFA destiné au lamido de Rey, par les éléments de la gendarmerie de Tcholliré et Poli ${ }^{11}$, laisse penser qu'il existerait une complicité entre les dogaris et les bandits. Ces accusations [...] semblent provenir du fait que le Baaba étant réputé quadriller son territoire, il paraît curieux que des bandes de dix à quinze personnes opèrent en toute impunité non loin des marchés de Mbaimboum, Touboro, Tcholliré et même jusqu'aux portes de Rey la capitale. Cela soulève en outre l'hypothèse de la sélection des renseignements fournis au Baaba: les dogaris agissant pour leur propre compte au nom mais à l'insu du souverain de Rey Bouba. [...] Dès lors, si l'on ne peut imputer directement la responsabilité de tout ce qui se passe au lamido, on est dubitatif sur le rapport des dogaris à l'insécurité. La dégradation considérable de la santé de Baaba amoindrit nécessairement sa capacité de contrôle et son emprise sur les faits et gestes de ses auxiliaires. En outre, le fait qu'entre 1999 et 2001 les interventions de l'armée contre les bandits aient fait des victimes parmi les dogaris de Rey Bouba tend à corroborer l'implication d'au moins une partie des dogaris dans le banditisme. »

Les dogaris sont l'œil et l'oreille du Baaba, ils sont le bras armé de son pouvoir et son pouvoir est un manteau dont ils recouvrent des agissements non commandés par le souverain. Certes, rien ne permet de supposer que les serviteurs du Baaba pourraient agir en dehors de ses ordres et pour leur propre compte, certes ils ne sont pas à l'abri de punitions et autres humiliations publiques. Mais Rey Bouba est un commandement vaste et c'est le zèle de ses serviteurs qui fait du Baaba le chef redouté qu'il est. Qu'ils soient membres de la cour et détenteurs d'un titre d'esclave-notable, qu'ils fassent partie de la milice du chef ou qu'ils soient en charge des travaux domestiques dans l'enceinte du palais, cette catégorie d'esclaves revendique son statut servile et le clame haut et fort. Pour ceux-là, l'affranchissement n'est pas envisageable parce que cela conduirait à la perte de leurs prérogatives. Le paradoxe de cette situation tient à ceci : pendant que l'autorité traditionnelle nie toute possession d'esclaves pourtant consubstantielle à son pouvoir, les esclaves quant à eux se définissent en tant que tels. En fait, une relation symbiotique lie le maître qui, officiellement, se

10. Terme par lequel on désigne les « coupeurs de routes », bandits de grand chemin qui tendent des embuscades sur les routes et dans les zones frontalières du NordCameroun.

11. Information publiée dans L'œil du Sahel, du 11 au 18 avril 2001. 
conforme au respect de la législation, aux serviteurs qui, en revanche, renoncent à la dignité d'homme libre en contrepartie d'une position sociale qui non seulement les met à l'abri du besoin, mais aussi et surtout les fait participer à la gestion du territoire. C'est pourquoi nombre d'observateurs de la dynamique historique de Rey Bouba estiment que le lamido est davantage le symbole du système politique de Rey Bouba que son véritable maître d'œuvre. C'est ainsi que le Baaba Abdoulaye Ahmadou, ancien député à l'Assemblée nationale, aurait voulu réformer le pouvoir de Rey Bouba lors de son intronisation en 1975. Or, il se ravisa très vite ou bien il en fut dissuadé, car au moment où il s'éteint, dans la nuit du 29 au 30 janvier 2004, c'est un pouvoir à l'apogée de son influence qu'il cède à son fils Moustapha.

Au demeurant, l'importance sociale prise par les esclaves ${ }^{12}$ royaux tend à corroborer l'expression de « revanche des maccube » qu'emploie Daniel Abwa (2002) pour exprimer le fait qu'à travers l'école et la participation au pouvoir traditionnel notamment, les peuples asservis ont finalement instrumentalisé l'ordre peul à leur profit. Nos interlocuteurs peuls ont parfois perçu l'arrogance et la brutalité des dogaris comme la renaissance de l'époque du bone ${ }^{13}$, celle des souffrances qui motivèrent leur adhésion au jihad d'Othman dan Fodio. Bien plus, les brassages par lesquels les Peuls ont incorporé les groupes conquis en les acculturant sont, a posteriori, vécus comme la manifestation pérenne de la trahison des chefs peuls envers la pureté ethnique peule. Par une sorte d'intégrisme culturel qui frise le racisme, d'aucuns, qui estiment être de «purs Peuls », confondent dans le même moule les dynasties des lamidats du Nord-Cameroun et les riimaybe («Peuls noirs » composés de descendants d'esclaves acculturés et souvent mariés à des femmes peules), puisque «du sang servile coule dans leurs veines », les chefs étant souvent nés de concubines kirdi. Aux yeux de certains Peuls, maccube (esclaves) et Kirdi sont pareils car, comme l'écrit Abwa, ce sont des gens asservis ou à asservir. Philip Burnham (1996 : 2223) partage aussi cet avis : " The term maccudo, when used in its broader sense, referred to all subject pagans regardless of their exact legal or social situation [...]. In practice, the Fulbe clearly viewed the vassal populations as functionally similar to slaves [...] the Fulbe state system was socially inclusivist in orientation but the defining logic of the Fulbe ethnic category remained exclusivist. » L'on perçoit là l'une des facettes de la question kirdi au

12. Les termes maccudo, jeyaado ou maraado par lesquels on désigne les personnes d'origine servile n'expriment qu'une idée de propriété, d'appartenance au patrimoine du maître plutôt qu'un quelconque euphémisme de dépendance ou de servitude envers celui-ci. Ce dernier détermine toujours sa relation avec «son esclave » en termes de propriété et non de clientélisme.

13. Bone (en fulfulde) signifie littéralement «souffrance». Historiquement, cette notion renvoie aux exactions et autres frustrations subies par les groupes de pasteurs peuls qui s'étaient installés pacifiquement dans le pays au XVIII ${ }^{\mathrm{e}}$ siècle. Les chefs kirdi exerçaient le jus de prima noctis sur les jeunes filles peules avant leur mariage. Les taxes et autres droits de pacage étaient fréquents, et l'assiette fiscale dépendait de l'humeur du chef de terre guiziga, moundang ou toupouri. Les vols de bétail étaient récurrents et impunis. 
Nord-Cameroun, ainsi que le débat généalogique qui, petit à petit, prend corps dans les conflits de positionnement consécutifs à l'ouverture démocratique.

Consubstantielles au système Ahidjo qui favorisa la primauté des valeurs sociales peules, la reproduction et l'instrumentalisation de la servitude connaissent des permanences et des ruptures selon que l'on vit en zone rurale ou en zone urbaine, selon que l'on a ou non accès à la bonne information religieuse et au débat sur les droits de l'Homme, selon le confort matériel et intellectuel. Relativement différente de l'Afrique de l'Ouest où la reproduction des classes s'apparente à l'intouchabilité atavique des personnes d'ascendance servile, l'évolution des statuts au Nord-Cameroun procède aussi bien des mutations générales de l'environnement politique et économique que des résistances individuelles.

\section{Maccube bilki'en ${ }^{14}$ : ignorance, indigence et servitude active en milieu rural}

Interrogé au sujet de la persistance de la servitude dans les relations sociales au Nord-Cameroun, Modibbo Abbo Hassoura Mbang répond :

«Même dans la charia islamique, l'esclavage est caduc. D'ailleurs, les razzias n'étaient pas précédées d'un appel à l'islamisation. Autrefois, le Peul était son propre maître et il possédait des esclaves. Le Blanc est venu posséder le Peul et ses esclaves. Puis l'onu nous a libérés des Blancs. Donc il n'y a plus d'esclaves. $\mathrm{Si}$ tu dis que tu possèdes des esclaves, tu mens. Celui qui nous possédait nous a tous libérés. Même l'Amérique a libéré les esclaves, à plus forte raison les Peuls » ${ }^{15}$.

À l'encontre de cet érudit de l'islam qui non seulement a consacré soixante-dix ans de sa vie aux Saintes Écritures, mais encore a voyagé et côtoyé l'administration et les colons français, se placent les marabouts qui, dans les villages, se font les gardiens du Temple. À travers un amalgame de prescriptions religieuses mal assimilées et de traditions opportunistes, le marabout recourait à l'islam pour encourager ses ouailles au paiement de la zakkat, l'aumône légale, en même temps qu'il justifiait la rétention de ce patrimoine par le chef traditionnel par le fait qu'il est le gestionnaire du baital mal, sorte de trésor public destiné à secourir les nécessiteux ou à accueillir les étrangers. Son discours sur la légalité de la servitude se fonde sur une lecture dogmatique du peu qu'il connaît de l'islam dont les codes juridiques sont présentés comme la justification des pratiques coutumières non islamiques :

«Le mouton égorgé par un esclave est impur à la consommation, la prière collective dirigée par un esclave est à recommencer, le pèlerinage à La Mecque effectué par un esclave sans le consentement de son propriétaire (jaumiiko) n'est pas différent

14. Littéralement, " esclaves idiots, ignorants, fermés ».

15. Modibbo Abbo Hassoura Mbang, entretien du 23 mai 2005, Ngaoundéré. 
d'une prière du vendredi. L'esclave n'a ni biens personnels, ni enfants, ni champ, ni même de sommeil car son propriétaire peut le réveiller quand il le veut pour faire ce qu'il lui demande de faire. Un esclave qui dort alors que son propriétaire est éveillé prend une grande option pour l'Enfer, car c'est par son propriétaire qu'il accède au Paradis tout comme c'est la satisfaction de l'époux qui donne le Paradis à la femme » ${ }^{16}$.

Ces propos émanent de quelqu'un qui fait office d'érudit dans un village, et que nous avons interviewé au sujet d'un litige qu'il avait tranché quelques années auparavant. Ce litige opposait les enfants d'un homme d'origine servile à ceux qui se présentaient comme ses propriétaires. Nanti de quelques biens, l'homme, sur son lit de mort, avait recommandé à ses enfants de faire don d'une partie de son patrimoine au chef; ce qui fut fait. Mais, en froid avec la famille dont il était le serviteur à cause d'une procédure d'affranchissement ayant viré à l'escroquerie, le défunt avait voulu évincer ladite famille de l'héritage en plaçant ses enfants sous la protection du chef traditionnel. Ce dernier, acculé, s'en remit au marabout du village, dans une sorte de ponce-pilatisme fort cynique. C'est ainsi que la progéniture du défunt s'entendit répondre : "Ces gens possèdent votre famille. Donc ces gens possèdent les biens de votre famille. Le conseil que je puis vous donner, c'est d'abandonner votre héritage en contrepartie de votre affranchissement. » Quant à la survie des enfants, «Dieu y pourvoira ».

$\mathrm{Si}$, sur le plan formel, l'esclavage fut aboli par le colonisateur et si le système carcéral fut abrogé dans les chefferies traditionnelles du NordCameroun, il demeure que le démantèlement réel de ces pratiques tarda à prendre effet. La résistance vint d'abord des chefs traditionnels qui, jusque dans les années 1930, continuèrent à perpétrer la traite grâce aux marges de manœuvre que le colonisateur allemand d'abord puis français, du temps des opérations de police, leur avait accordées dans la «pacification » du pays kirdi. Aux rapts s'ajouta la vente proprement dite, celle-là concernant surtout des individus que les chefs, tels Mangala, chef du canton de Douvangar, vendaient aux Peuls en guise de condamnation pour adultère, vol ou inceste. Dans la seule circonscription de Mokolo, cinquante-sept condamnations furent prononcées en 1933 et vingt-sept en 1934 pour « faits de traite ». Les lamidats de Baschéo et de Demsa constituaient alors de véritables plaques tournantes de la traite transfrontalière entre le Cameroun sous administration française et le Nigeria britannique ${ }^{17}$.

Le second facteur favorable à la reproduction des pratiques esclavagistes tient à la nature de la «politique nordiste » du président Ahmadou Ahidjo. Ainsi, jusqu'en $1977^{18}$, Ahidjo avait laissé les mains libres aux autorités

16. Interview du 27 décembre 2004, Dargala.

17. Archives nationales de Yaoundé, APA $11763 / \mathrm{M}$. Lettre $\mathrm{n}^{\circ} 56 \mathrm{f}$ du chef de la Circonscription de Garoua, 20 février 1935.

18. Le décret $\mathrm{n}^{\circ} 77 / 245$ du 15 juillet 1977, portant sur l'organisation des chefferies traditionnelles, réforme cette institution dans le sens de la réduction des pouvoirs des autorités traditionnelles. Ces dernières deviennent des « auxiliaires de l'admi- 
traditionnelles dans la gestion de la périphérie, en contrepartie d'une allégeance sans faille au pouvoir central. C'était le prix à payer dans un pays alors secoué par de multiples forces centrifuges (Fogui 1990). L'exercice de l'autorité par les chefs traditionnels prit parfois les allures d'une véritable substitution de l'autorité traditionnelle à l'autorité administrative, car en collectant l'impôt, en «arrêtant» les enfants en âge scolaire, en présidant parfois aux destinées des sections locales du parti unique, les chefs disposaient de tellement de moyens de contrainte sur les populations et d'influence sur les représentants de l'État que l'on pouvait avoir l'impression qu'ils avaient été restaurés dans la plupart de leurs prérogatives d'antan. Une mission d'inspection administrative dénonça d'ailleurs cette situation dans un rapport qui accusait la politique adoptée envers les chefs d'aller à contre-courant de la modernisation, car concourant à l'immobilisme ${ }^{19}$.

Au-delà de la realpolitik qui inspira la «compréhension» du pouvoir central envers les autorités périphériques, l'on est fondé à penser que cela relevait, de la part d'Ahidjo, d'un compromis personnel. En effet, Ahidjo n'avait pas été accepté, mais simplement toléré par les chefs qui, tout au long de son ascension politique dans le contexte de la décolonisation, lui reprochèrent ses origines roturières. Non seulement il n'était pas issu de l'aristocratie traditionnelle, mais encore on le disait de «père inconnu », un euphémisme dont d'aucuns font l'économie pour le traiter de «bâtard ${ }^{20}$. Dans l'obscurantisme qui caractérisait le Nord-Cameroun à l'aube des indépendances et qui persiste d'ailleurs dans nombre de zones rurales, l'enfant naturel était en réalité moins bien loti que l'esclave, car il était réputé être physiquement intouchable, génétiquement impur. On croyait que tout contact physique avec un enfant naturel entraînerait les brûlures atroces du feu de la géhenne à l'endroit du corps où s'effectuerait le contact. Si Ahidjo ne vécut pas nécessairement les affres de sa filiation jugée incertaine, il reste que le discours tout comme l'attitude des chefs traditionnels exhalaient le mépris. Si, malgré tout, Ahidjo se hissa au sommet de l'État camerounais, il n'osa pas aller dans le sens du modernisme et des droits de l'Homme et laissa libre cours à l'autorité des chefs dans leurs commandements respectifs. Aller à leur encontre, était entreprendre un combat perdu d'avance qui

nistration » (article 20), bref, des fonctionnaires qui ont droit à un salaire, mais peuvent subir des sanctions administratives.

19. 1 AA 1378, Rapport de synthèse des travaux de la commission d'inspection dans le département du Logone et Chari du 5 février au 7 mars 1969.

20. Au sujet du débat sur la filiation d'Ahmadou Ahidjo, voir Philippe GaILlard (1994) et Maimounatou (2002). La version des faits de Maimounatou est bien différente de celle de DaKolé Daïssala (1993 : 31) qui écrit : «L'homme public Ahidjo se devait d'avoir plus d'une corde à son arc pour pouvoir s'imposer à un Nord-Cameroun soupçonneux à son égard [...]. Fruit du hasard, sans références, Ahidjo était suffisamment intelligent pour se savoir vulnérable, "inacceptable" pour ce Nord sahélien aux traditions socio-politiques séculaires, carrées, avec un système de dévolution du pouvoir précis, avec une conception de la légitimité irréductible [...] et ce dans le cadre d'un patriarcat rigide et intransigeant. » 
ne manquerait pas de fragiliser l'alliance conclue avec les chefs. Dès lors, la servitude persista. Le colonisateur tout comme les administrateurs du Cameroun indépendant les appelaient "serviteurs », boys ou domestiques, mais hormis la vente, ces individus étaient manifestement des captifs qui ne pouvaient se défaire de leurs liens sans représailles. Les chefs eux-mêmes les désignaient par le terme de suka'en qui signifie aussi bien jeunes gens athlétiques que dépendants, clients liés à la personne d'un patron dont ils exécutent les tâches subalternes et autres corvées.

Dans ce contexte, l'on vit se perpétuer les travaux forcés sous l'avatar de la main-d'œuvre champêtre et sous la forme de prestations qui n'avaient rien à envier aux pratiques coloniales. Dans les villages, le travail de l'esclave se partageait entre son propre lopin de terre et la plantation de son maître, car l'esclave ne pouvait ni accéder à la propriété foncière, ni disposer d'un champ de grande superficie. Si, exceptionnellement, il parvenait à disposer d'un grand champ, il devait partager sa récolte avec son maître, lequel, au moment voulu, pouvait se servir dans son grenier. Lorsque le crieur public invitait la population à converger vers la plantation du chef ou à entretenir les routes, les maîtres se faisaient représenter par leurs esclaves. Avant la visite des chefs d'États étrangers, qu'Ahidjo recevait souvent à Garoua ou à Maroua, la main-d'œuvre servile était mise à contribution pour aménager les lieux d'accueil où viendrait séjourner par la suite la délégation du canton ou du lamidat. Une telle atmosphère de hiérarchisation sociale fit le lit de bien des drames personnels.

\section{Damdam, l'élève servile}

L'école d'un petit village de la plaine du Diamaré abritait à la fin des années 1960 une cinquantaine d'enfants. Le maitre, M. Gaston, était originaire de la partie méridionale du pays. L'homme avait la réputation d'être un enseignant autoritaire, ami des élèves brillants, terreur des paresseux. Chez lui, le chef de classe devait toujours être l'élève qui donnait l'exemple par son assiduité et surtout ses résultats scolaires. Dans la semaine, il y avait un jour spécifique où M. Gaston portait sa chemise rouge à col Mao. Ce jourlà, il y avait « dictée-questions » ou «Coran » (récitation «par cœur» du cours précédent de sciences d'observation, d'histoire, de géographie ou d'instruction civique). La «formule pédagogique » était simple : l'élève qui avait «zéro faute» en dictée ou qui récitait toute la leçon sans bégayer devait fouetter le reste de la classe, au prorata du nombre de fautes inventoriées dans le cahier de chacun. Damdam était la meilleure élève de la classe. Damdam était chef de classe. Damdam fouettait donc la majorité des élèves tout le temps, s'appliquant avec soin sur le dos des garçons. Avoir une fille chef de classe était déjà assez difficile à supporter ; en recréation, les garçons des autres classes prenaient un malin plaisir à railler leurs camarades de la classe de M. Gaston, qui acceptaient d'être «tapés» par une fille. 
D'aucuns, semble-t-il, y avaient trouvé une source de motivation pour apprendre leurs leçons, par fierté. Et M. Gaston savait tenir compte des progrès réalisés par ses élèves, en les dispensant de temps à autre du fouet. Cela encouragea nombre d'élèves, mais pas Moustapha. Il était le yérima, le prince, le fils du chef de village.

Évidemment il était au-dessus du fouet. Pire, il avait mal à l'âme de devoir courber l'échine devant Damdam, car, se remémora-t-il au cours de notre conversation «c'était comme si la bicyclette enfourchait le cycliste.» Moustapha se plaignit auprès de Sa Majesté son père, qui ne comprenait pas comment il était possible qu'une $k o r d o^{21}$ s'autorise un tel acte de lèsemajesté. Damdam raconte que son année scolaire fut empreinte de brimades, d'injures, de corvées d'utilité publique auxquelles son père fut régulièrement convié avec insistance. À la fin de l'année scolaire, son père fut «prié » de la retirer de l'école au prétexte qu'elle faisait la «bordellerie ». Damdam quitta le village et se rendit auprès d'un parent résidant en ville. Là, elle poursuivit ses études primaires et obtint le Certificat d'études primaires élémentaires, ce qui lui permit de suivre une formation d'aide-soignante et de devenir une mère très attachée à l'instruction de ses enfants. Damdam aime à rappeler que Moustapha se déplace toujours à bicyclette, porte un boubou délavé et habite une case au toit de chaume ${ }^{22}$.

\section{Aminou Sadjo et la leçon d'histoire fatale}

Tout comme Damdam, un ancien élève d'une école de village devenu opérateur économique dans la ville de Garoua se souvient de la leçon d'histoire au cours élémentaire deuxième année, qui lui coûta sa scolarité en 1980. Le maître, un Peul originaire des environs de Maroua, avait l'habitude de prendre des exemples alentours pour étayer ses explications. Les sujets de rédaction portaient toujours sur les gens du voisinage, le marché d'à côté. En calcul, l'on additionnait et multipliait du bétail, des beignets, de l'argent. En sciences d'observation, on observait réellement. Ses anciens élèves sont unanimes pour dire que les leçons d'histoire constituaient des régals, car le maître se mettait dans la peau du conquérant peul pour parler des guerres précoloniales, accompagnait les explications d'histoires croustillantes, jouait vraiment le personnage là où cela était possible.

Un jour, la leçon d'histoire porta sur le commerce des esclaves. Le maître apporta des cordes en classe et invita des élèves à se les passer au cou, il parla des marchands d'esclaves, mais aussi des noms que l'on donnait aux nouveau-nés d'origine servile. Il cita Sadjo entre autres noms. Aminou, fils de Sadjo, se rappelle encore cette leçon d'histoire qui lui attira des

21. Kordo en fulfulde est le féminin de maccudo, esclave, et s'oppose à dimo, personne de naissance libre.

22. Interview du 30 décembre 2004, Maroua. 
problèmes, car en classe, certains de ses camarades ne voulaient plus s'asseoir aux côtés d'un fils d'esclave, d'aucuns l'évitaient dans la cour de récréation. Ainsi, il finit par abandonner l'école ${ }^{23}$. Comme bon nombre de musulmans du Nord-Cameroun, ses enfants portent désormais des noms arabes afin de paraître plus musulmans, peut-être aussi pour faire moins esclaves ${ }^{24}$.

$\mathrm{Au}$ demeurant, plus que la nature sahélienne du Nord-Cameroun, c'est davantage le retour des chefs traditionnels sur la scène sociopolitique et le maintien subséquent des pesanteurs socioculturelles qui favorisèrent la persistance de l'exploitation des personnes d'origine servile. Illégitime du point de vue de sa justification religieuse et illégale dès lors que cette coutume d'un autre âge a été abolie par le colonisateur, la servitude, pour ne pas dire simplement l'esclavage, a survécu à l'indépendance, avec des manifestations quotidiennes dans les zones rurales. Mais, au-delà de l'appétence des chefs traditionnels pour une pratique consubstantielle à l'apparat du pouvoir, d'une part, et l'importance que lui accordent les maîtres, d'autre part, pour des raisons tantôt économiques tantôt de prestige, la persistance de formes larvées de servitude d'un autre âge procède aussi de la résignation des personnes d'origine servile dont la contraction de l'horizon mental réduit à la sphère du village et de ses environs, les enferme dans des attitudes de marginalité.

Fruit de l'ignorance, de la pauvreté et de l'enfermement moral, la servitude dans les zones rurales est un exemple d'encasernement psychologique : parfois même, l'esclave croit accéder au paradis céleste en se pliant pendant des décennies aux moindres caprices de celui qu'il considère comme son propriétaire; souvent, il espère un affranchissement pour services rendus. Par exemple, dans nombre de villages de l'Extrême-Nord où le seuil de pauvreté traduit plutôt paradoxalement un meilleur niveau de vie pour des populations manifestement indigentes, le fruit du labeur quotidien de certaines personnes leur sert à négocier leur affranchissement, durant des décennies, car elles espèrent naïvement que celui-ci effacera leur différence. Or, dans les villages, la différence reste intrinsèque à l'ascendance servile et même si les stigmates sociaux sont moins marqués qu'en Mauritanie ou au Niger, les conclusions de Boubacar Messaoud que reprend Roger Botte (2000a : 12) s'appliquent au Nord-Cameroun dans le sens où : "Même l'affranchissement n'est pas une dimension de la liberté mais au contraire une dimension de l'esclavage. » Quelques exemples permettent d'apprécier à quel point asservissement et affranchissement peuvent être les revers transparents d'une même médaille.

23. Interview du 3 janvier 2005, Garoua.

24. Sur le snobisme qui entoure le baptême des nouveau-nés dans la partie septentrionale du Cameroun, voir Hamadou Adama (1998). 
Salaire mensuel contre promesse d'affranchissement: douze ans d'escroquerie

Yacoubou était un jeune fonctionnaire moyen qui, en 1978, venait de recevoir son premier salaire en même temps que le rappel des mois précédents qui n'avaient pas encore été pris en compte. Muni de 500000 francs CFA (environ 750 euros), il se rendit auprès d'un homme racheter la « liberté » de sa mère, qu'elle rêvait d'obtenir avant sa mort. Le maître de celle-ci le reçut avec beaucoup de courtoisie, fit rôtir un poulet - ce qui est une marque de considération au village - et le remercia infiniment pour tout cet argent qu'il lui offrait. Il lui promit de faire le nécessaire, mais il fallait d'abord qu'il consultât ses frères et sœurs, car bien qu'étant l'aîné des héritiers de la mère et de sa progéniture, il ne pouvait prendre seul cette décision. Les jours passèrent, puis les semaines, puis deux mois. Yacoubou s'en retourna demander la suite. Il reçut la réponse suivante : «J'attendais de voir comment tu agirais à la fin du mois, quand tu auras pris ton salaire, si tu viendrais me saluer. Tu n'es pas venu, ce qui veut dire qu'une fois ta mère affranchie, je ne te verrai plus. »Yacoubou nous confia que ce fut le point de départ d'un chantage : pris entre les exigences intarissables du maître et la sourde pression de l'amertume maternelle, Yacoubou dut budgétiser le maître de sa mère dans son salaire mensuel afin de lui verser environ le tiers de ses émoluments. Cela dura douze ans, douze ans d'escroquerie car, à la mort du maître, en 1990, Yacoubou apprit de ses proches qu'il ne fut jamais dans ses projets d'affranchir un esclave. Pire, le maître considérait l'argent qu'il recevait comme une sorte de compensation dans la mesure où il ne pouvait faire travailler Yacoubou d'une autre manière pour son compte $^{25}$.

Des esclavagistes de ce type se terrent encore dans des villages, arrivant chez des personnes qu'ils appellent pudiquement bandiraabe (parents), pour exposer des besoins en mil, en argent, en vêtements, jouant de la division au sein d'une même famille. En effet, des cas existent où certains membres âgés de la famille ont été affranchis de la propre initiative du maître, tandis que les autres membres devront, de longues années durant, entretenir un oisif vivant de leur travail en raison de l'empreinte psychologique qui marque leurs esprits. La frange affranchie se fait curieusement amnésique des longues années d'assujettissement, de privations et enseigne la patience et la subordination aux desiderata du maître. Or, l'affranchissement dans ce cas de figure, celle d'une personne âgée, ne signifie rien d'autre que le dégagement d'une racine morte, dont le suc aura été sucé pendant des années, une loque humaine, désormais, exprimant encore sa gratitude pendant le peu de temps qui lui reste à vivre... en manifestant sa disponibilité au maître en cas de besoin. D'aucuns, parmi les jeunes, trouvent une sorte de salut dans l'exode vers la ville où s'élabore, au contact de la modernité,

25. Interview du 30 décembre 2004, Maroua. 
un discours qui passe pour être révolutionnaire. Le nouveau citadin rentre dans une communauté cosmopolite au sein de laquelle il parvient implicitement à comprendre l'iniquité de son statut. À ce propos, le feuilleton télévisé Racines d'Alex Haley que diffusait la télévision camerounaise contribua, aux dires de certains de nos interlocuteurs, à leur faire accepter le passé servile de leurs parents, car «même les stars noires d'Amérique sont des descendants d'esclaves ». Toutefois, Racines fut aussi un vecteur de l'insoumission du jeune esclave averti, lequel pouvait défier le maître de ses parents en l'ignorant tout simplement. Mais même l'exode urbain reste, pour une personne d'ascendance servile, une sorte d'exil puisqu'il lui a fallu partir pour exister. Naguère, l'esclave extrait de son milieu se retrouvait dans un environnement nouveau où il endossait une nouvelle identité culturelle au contact d'autres esclaves et au contact de la culture dominante. Séparé de sa famille d'origine, il n'avait plus de parents autres que sa descendance, car la razzia, la vente et la déportation disloquaient des familles dont les membres étaient souvent à jamais séparés les uns des autres. En ville aussi, c'est une nouvelle famille qui se crée. Avec le temps, les gens se fondent dans l'anonymat de la ville et fréquentent de moins en moins leur village d'origine ; à la mort de leurs parents, ils s'éloignent parfois des oncles, cousins et autres tantes restés dans un village où le stigmate servile les accable pour toujours.

\section{Perdo maratake ${ }^{26}$ : savoir, avoir et " revanche des maccube»}

Introduite au Nord-Cameroun avec la colonisation, l'École occidentale tarda cependant à s'y implanter. Dans la plupart des cas, c'est au lendemain de la Seconde Guerre mondiale et souvent dans les années 1950 que les écoles publiques à cycle complet atteignirent les grands villages. Avant la fin des années 1970, rares étaient les arrondissements disposant d'un collège. En dépit de cette médiocre couverture scolaire, la fréquentation de l'École occidentale s'accrut. Contrairement à l'affirmation véhiculée par nombre d'écrits relatifs à l'histoire de l'École dans cette partie du Cameroun, l'aristocratie traditionnelle ne se détourna pas systématiquement de l'École, symbole d'acculturation. Et pour cause. Les jeunes princes se rendaient à l'école, accompagnés d'un serviteur ; ce dernier restait assis à la porte d'entrée de la classe pendant que son jeune maître suivait, quoique souvent distraitement, les leçons. Il importait seulement au colonisateur que ses futurs collaborateurs acquissent des rudiments de français pour faciliter la communication, mais aussi l'assimilation.

26. L'expression signifie en fulfulde que l'on ne peut asservir un individu « civilisé ». Emprunté au vocabulaire colonial et synonyme d' «évolué », le terme "civilisé » s'applique, dans le jargon local, aux personnes instruites, à ceux qui ont « voyagé », bref, aux gens qui ont une allure citadine, vont à la mosquée en jeans et chemisette, lisent des romans, etc. Perdo peut connoter, selon le contexte, l'idée d'être « clairvoyant », « futé »... 
C'est donc assis à l'entrée de la classe ou collés à la fenêtre que les serviteurs-accompagnateurs captèrent un peu de français et entrèrent de cette manière dans les rouages du système colonial, lequel les employa comme messagers, porteurs d'ordres, et recommanda leur maintien dans l'entourage du lamido. C'est à eux que revenait le titre d'adjia, d'intermédiaire entre l'autorité coloniale et l'autorité traditionnelle. La fonction, par la suite, fut entérinée par l'administration postcoloniale. C'est généralement parmi la progéniture des serviteurs des grandes autorités traditionnelles que l'on recense les lettrés d'ascendance servile, ceux qui poussèrent les études audelà du certificat de fin d'études primaires, ce premier diplôme ouvrant d'ailleurs les portes de l'administration publique à une époque où ministres, préfets, sous-préfets et députés originaires du Nord-Cameroun possédaient rarement plus que ce parchemin. Mais à une période où l'accès au pouvoir et aux hautes fonctions dans l'administration publique se jouait surtout - mais pas seulement — entre les fonctionnaires peuls et la progéniture des chefs traditionnels, les autres prétendants, qu'ils fussent ou non d'ascendance servile, devaient se contenter des métiers techniques et de services tels que l'enseignement, la santé, l'élevage et l'agriculture.

Comme, contrairement au Niger (Botte 2000b), les maîtres ne s'opposèrent pas en général à l'instruction de leurs esclaves, ces derniers envoyèrent leurs enfants à l'école publique qui était alors gratuite, car l'État procurait livres, cahiers, médicaments et même quelques aliments aux élèves. En outre, une politique d' " arrestation » des enfants en âge scolaire fut appliquée jusqu'au milieu des années 1970 pour les obliger à rejoindre les bancs. Des tournées de sensibilisation effectuées par les responsables du parti unique dans les villages du Diamaré invitaient les populations à envoyer les enfants à l'école; à l'occasion, on présentait triomphalement à la foule un chercheur débutant comme «un savant» et il devenait l'exemple à suivre. Gratuité de l'école, sensibilisation et perspectives de mobilité sociale favorisèrent donc l'instruction des gens du commun. Une instruction cependant limitée par les difficultés financières avec le désengagement progressif de l'État et l'appel à la contribution des parents à l'éducation de leurs enfants.

L'instruction jeta les fondements d'un affranchissement tacite. Progressivement, et sans qu'il y ait nécessairement une relation de cause à effet entre l'arrivée d'un chrétien sudiste au pouvoir en 1982 et le relâchement des rapports de servitude, la remise en cause du «système Ahidjo » ne fut pas sans impact sur la remise en cause, au plan individuel, des pratiques socioculturelles discriminatoires. Certes, des chefs traditionnels se sont orientés dans le sens de l'histoire en accompagnant l'ascension sociale, voire politique, des descendants de leurs serviteurs ou de ceux de leurs prédécesseurs ; d'ailleurs, cette attitude prend de l'importance dans des contrées où se nouent des partenariats entre les intellectuels d'origine servile et les détenteurs du pouvoir traditionnel, dès lors que la formation professionnelle 
et l'engagement politique assurent une meilleure réussite sociale. Néanmoins, médecins, ingénieurs, enseignants et autres d'ascendance servile ont repris à leur compte le nouveau contexte pour sensibiliser leurs proches sur l'iniquité des liens qui entravent leurs mentalités. Porteur avec les plus jeunes, leur discours bute sur l'opacité de l'esprit des plus anciens. Un jeune homme vivant en Europe posa la question suivante à sa grand-mère : « Pourquoi persistes-tu à vouloir nous léguer cette tare ?» Il reçut la réponse suivante : « Tu as changé de pays, veux-tu aussi changer de grand-mère?», comme pour dire qu'il doit se résigner à son ascendance servile ${ }^{27}$. En revanche, son frère cadet a rejeté la résignation qui habite l'esprit de la grand-mère conditionnée par des décennies de servitude. Cet étudiant en droit nous confia qu'il est «le Lance Armstrong de l'esclavage; ce gars a vaincu le cancer qui rongeait son corps, moi je vais vaincre les esclavagistes qui sont le cancer du Nord-Cameroun $»^{28}$.

Chez nombre de nos interlocuteurs intellectuels, la tendance est au mépris envers ceux qu'ils ne perçoivent plus désormais que comme des « profiteurs », des « exploiteurs », des « oisifs », des «nobles mendiants », des «mécréants » pour ne citer que ces expressions parmi les moins dépréciatives, sinon obscènes. À la rage contenue des présumés maîtres dont quelques-uns ont accepté de réagir à quelques-unes de nos questions, répond une certaine tendance à la provocation parmi les intellectuels d'ascendance servile. Il s'agit de « pousser à la faute ${ }^{29}$ celui qu'on ne présente plus que comme l' «adversaire », pour qu'il soit pris en «flagrant délit de pratique esclavagiste ». Alors, « il sera entendu par la police, la gendarmerie, l'huissier pour qu'il explique les tenants et les aboutissants de son droit de propriété, qu'il dise quand, comment, où et dans quelle circonstance nos aïeux sont devenus les esclaves de ses aïeux ».

Chez les opérateurs économiques d'origine servile, l'initiative de l'affranchissement est souvent venue du maître, surtout lorsqu'il est peu nanti comme c'est fréquemment le cas. Nous n'avons pas pu recueillir les témoignages des concernés eux-mêmes, mais dans leur entourage à Maroua, Garoua et ailleurs dans les centres urbains du Nord-Cameroun, des causeries informelles nous ont permis de mettre au jour les procédés par lesquels des maîtres ont offert l'affranchissement ${ }^{30}$ pour, en réalité, entrer dans les

27. Interview, Yaoundé, avril 2005.

28. Interview, Yaoundé, avril 2005.

29. Il s'agit par exemple de faire constater par un huissier de justice des attitudes esclavagistes (réclamation d'argent ou de quote-part d'héritage, brimades, négociation de l'affranchissement), voire de susciter une dispute qui dégénérerait en affrontement physique avec le maître ; l'intervention des forces de l'ordre permettant alors d'ouvrir une information judiciaire.

30. Il peut y avoir autant d'affranchis que de modes d'affranchissement. Lorsque c'est le maître qui libère (du verbe yoofugo, en fulfulde «libérer »; rimdingo, affranchir) une esclave, il convoque son mari et prononce la déclaration d'affranchissement en présence d'un témoin honorable (imam, chef de quartier, etc.). Lorsque le maître libère un homme, celui-ci reçoit lui-même la déclaration d'affranchissement en présence d'un ou de plusieurs témoins. Parfois, c'est un 
bonnes grâces de tel homme ou de telle femme que la fortune a hissé au sommet de la hiérarchie sociale. C'est quelquefois la famine au ventre et la peur à la gorge qu'ils ont sollicité une audience. Assis à même le sol, vêtus de haillons, la peau marquée par les intempéries de la vie, articulant péniblement leurs mots, ils ont déclaré que rien, sinon des liens familiaux, ne les lient désormais à leur interlocuteur et à ses ascendants serviles. Des descendants, il n'est pas question puisque les nantis épousent généralement des femmes d'origine libre. L'affranchissement des nantis ne s'effectue cependant pas aussi facilement dans tous les cas, car il faut parfois compter en millions de francs CFA pour que d'aucuns acquièrent leur nouveau statut d'hommes libres. Dans tous les cas, l'affranchissement, octroyé ou acheté, entraîne soit la prise en charge pérenne de l'ancien maître, soit le don d'un fonds de commerce qui lui permettra d'entreprendre une activité génératrice de revenus. Dans un cas comme dans l'autre, on s'inscrit dans une logique d'exploitation.

Au demeurant, la diffusion de Racines et des feuilletons brésiliens par la télévision camerounaise, l'ouverture démocratique et la prolifération des organismes de défense des droits de l'Homme, le commerce et l'accès à l'État, des films tels que Malcom $X$ et bien d'autres facteurs de changement des mentalités ont eu des incidences sur le comportement aussi bien des esclaves que des maîtres. Implicitement, les liens de servitude se relâchent.

Le premier constat à la lumière de l'analyse et des témoignages qui précèdent est celui de la rémanence de la condition servile dans la partie septentrionale du pays. Celle-ci revêt plusieurs dimensions : d'abord, l'esclavage proprement dit n'a pas disparu avec l'accession du Cameroun à l'indépendance. Se fondant indûment sur l'islam et la crédulité de leurs victimes, des personnes se présentant comme « propriétaires » ou «maîtres » jouissent sans contrepartie du labeur d'autres personnes en vertu de liens historiques hérités de leurs parents et qu'elles entendent parfois transmettre à leur descendance. Ensuite, la servitude marquée par des liens de subordination matériellement diffus, mais psychologiquement contraignants, perdure. Enfin, le stigmate servile, même après des décennies d'affranchissement, reste rivé à vie sur l'individu.

intermédiaire (commerçant, député...) qui intercède auprès du maître pour un affranchissement qui coûte une centaine de milliers de francs CFA (150 euros) dans les villages. On en parle exceptionnellement à la mosquée, le muezzin informant les fidèles après la prière de l'aube (fadjr) ou de la nuit (icha), à des heures où la mosquée est peu remplie et accueille généralement les personnes âgées, discrètes. Les autres l'apprendront par ouï-dire ou le découvriront le jour de la fête du sacrifice quand l'affranchi égorgera lui-même son mouton. L'affranchissement, tout comme l'asservissement, s'effectue donc dans l'informel. 
Le deuxième constat qui s'impose est celui de la survivance de l'esclavage royal. Une forme altérée, semblable à la domesticité, se prolonge de manière consubstantielle dans toutes les chefferies traditionnelles du NordCameroun. Toutefois, dans le lamidat de Rey Bouba, s'éternise une économie de la soumission qui offre aux esclaves une marge de manœuvre inouïe dans l'administration et l'exploitation du territoire. Cependant, même là, on note une édulcoration du système depuis la mort du lamido Abdoulaye Ahmadou en 2003. Qu'en adviendra-t-il dans les années à venir ? Les dignitaires serviles lâcheront-ils du lest ou bien réussiront-ils à préserver l' « intégrité politique et territoriale » de Rey Bouba comme ce fut le cas en 1975 à l'avènement du lamido Abdoulaye ?

Le troisième constat met en évidence l'émergence d'une stratégie de la confrontation et de reniement des liens serviles parmi les jeunes générations d'intellectuels, qui se substitue à la négociation dans les procédures d'affranchissement. Une stratégie efficace dans les villages comme en ville. D'une part, elle implique des affranchissements par renonciation du maître ou négociés sans délais; comme dans un élan de générosité, le maître octroie gracieusement la liberté ou alors ne traîne plus pour prononcer l'affranchissement lorsque l'esclave sollicite le rachat de sa liberté. D'autre part, on enregistre des cas de rupture unilatérale des liens de servitude; désormais, l'esclave ignore le maître et interdit à ses proches tout lien social avec sa famille (notamment lors des cérémonies de deuil, de mariage ou de baptême).

Le quatrième constat consiste à souligner le lien que l'on est fondé à établir, à la suite de Daniel Abwa, entre la problématique de la kirditude et celle de la servitude. En effet, au-delà de l'asservissement et de la marginalisation que l'auteur présente comme le dénominateur commun aux Kirdi et aux maccube, se profile un débat souterrain, celui de l'identité de l'esclave et de l'identité du maître, d'une part, et, celui de l'identité peule ou arabe, d'autre part. Historiquement, les esclavagistes étaient aussi bien musulmans qu'animistes puisque les razzias et la traite des esclaves n'étaient pas le fait des seuls pouvoirs centralisés musulmans. En théorie, tous les esclaves provenaient des sociétés kirdi ; mais, très tôt islamisés et incorporés dans la culture dominante, ils ont parfois acquis à travers le savoir islamique et les mariages les traits physiques et culturels peuls, kanouri, arabes ou mandara. Si ailleurs dans le pays l'intégrité ethnique n'est pas un sujet de débat, tel n'est pas le cas des sociétés peule et arabe du Nord-Cameroun où les commérages sur l'ascendance de tel homme politique ou les origines de tel homme d'affaires visent à prouver sa « fulanité douteuse » ou son « arabité imparfaite », comme si la traçabilité de la «pureté ethnique » était possible dans un Nord-Cameroun multiculturel. Paradoxalement, c'est à la société peule elle-même que ce débat nuit le plus, divisant parfois des familles. On assiste, hélas, à de vaines tentatives pour s'arrimer à une souche peule « authentique » de la part de « Peuls noirs » nécessairement hybrides. « Assumons chacun notre part de kirditude ou de servitude et tournons la page, car à force de fouiller les tombes pour retrouver Chehou Oussoumanou ou Oqba 
ben $\mathrm{Nafi}^{31}$, on risque de croiser des chenilles », déclare, sans convaincre, un jeune prince peul conscient des réalités génétiques de l'aristocratie du Nord-Cameroun.

Quoi qu'il en soit, et ce sera notre cinquième constat, pendant que la descendance des anciens maîtres s'assombrissait du fait du recours immodéré aux concubines guiziga, moundang, fali, gbaya ou mboum, celle des anciens esclaves, surtout nantis, s'éclaircissait du fait du recours aux épouses peules. Le noir n'est plus le teint homologué de l'esclave, tout comme la finesse des traits physiques n'est plus un gage de fulanité. D'ailleurs, le discours sur la servitude et la sexualité se révèle si riche de renseignements qu'il ébranle bien des certitudes sur le mariage et l'honneur.

En définitive, au Cameroun, l'esclavage et ses dérivés archaïques ne posent pas un problème de relation à l'État car, sur le plan formel, ces pratiques sont abolies. L'État a brisé les liens de servitude, mais le maintien des normes socioculturelles précoloniales et l'ignorance ont reproduit les liens d'exploitation. Des liens que le savoir et l'avoir brisent sur le plan individuel, en attendant qu'un incident vienne provoquer un débat public.

Département d'Histoire, Université de Ngaoundéré, Cameroun.

\section{BIBLIOGRAPHIE}

\section{ABDOUl KADIRI}

2002 La dynamique de l'institution militaire dans les lamidats du Nord-Cameroun (XIX ${ }^{e}-X X^{e}$ siècles) : cas de Rey-Bouba et de Ngaoundéré, Mémoire de maîtrise, Ngaoundéré, Université de Ngaoundéré.

ABwa, D.

2002 «La revanche des maccube du Nord-Cameroun», Communication au deuxième séminaire de la FALSH, Yaoundé Université de Yaoundé-I, inédit.

BAH, T. M.

1982 «Les armées peul de l'Adamaoua», Études Africaines offertes à Henri Brunschwig, Paris, Éditions de l'EHESS.

1993 «Le facteur peul et les relations inter-ethniques dans l'Adamaoua au $\mathrm{XIX}^{\mathrm{e}}$ siècle », in H. Adala \& J. Boutrais (dir.), Peuples et cultures de l'Adamaoua (Cameroun), Paris, ORSTOM : 61-86.

BALES, K.

2004 [1999] Disposable People. New Slavery in the Global Economy, Berkeley University of California Press.

31. Respectivement initiateur du jihad peul qui a abouti à la conquête d'une bonne partie du Nord-Cameroun et ancêtre présumé des Peuls, d'origine yéménite. 
BARTH, H.

1965 [1857] Travels and Discoveries in North and Central Africa being a Journal of an Expedition Undertaken Under the Auspices of HBM's Government in the Years 1849-1853, vol. II, Centenary Edition, London, Frank Cass.

BURNHAM, P.

1996 The Politics of Cultural Difference in Northern Cameroon, Edingburg, Edingburg University Press.

BotTe, R.

2000a «De l'esclavage et du daltonisme dans les sciences sociales », Journal des africanistes, 70 (1-2) : 7-42.

$2000 \mathrm{~b}$ «Le droit contre l'esclavage au Niger», Politique africaine, 90 : 127-143.

DAKOLÉ DAÏSSALA

1993 Libre derrière les barreaux, Paris, Jaguar.

De Garine, I.

1964 Les Massa du Cameroun : vie économique et sociale, Paris, PUF.

Denham, D., Clapperton, H. \& Oudney, W.

1826 Voyages et découvertes dans le Nord et dans les parties centrales de l'Afrique..., exécutés pendant les années 1822, 1823 et 1824, vol. II, Paris, Arthus Bertrand.

Fogui, J.-P.

1990 L'intégration politique au Cameroun, une analyse centre-périphérie, Paris, LGDJ.

GAILLARD, P.

1994 Ahmadou Ahidjo. Patriote et despote, bâtisseur de l'État camerounais, Paris, Jalivres.

HAMAdOU AdAma

1998 «Les nouveaux prénoms des Peuls du Nord-Cameroun», Islam et sociétés au sud du Sahara, 11: 67-84.

IBn Fartua, A.

1970 History of the First Twelve Years of the Reign of Mai Idris Aloma of Bornu (1571-1583) Together with the Diwan of the Sultans of Bornu and Girgam of Magumi, London, Frank Cass and Co. Ltd.

LENFANT, E.

1905 La grande route du Tchad, Paris, Hachette.

LOVEJoy, P. E.

2000 Transformations in Slavery: A History of Slavery in Africa, Cambridge, Cambridge University Press. 


\section{MaimounAtou}

2002 Le tremplin politique de Ahmadou Ahidjo au Nord-Cameroun : 1946-1960, Mémoire de maîtrise, Ngaoundéré, Université de Ngaoundéré.

NACHTigal, G.

1980 Sahara and Sudan. Vol. II. Kawar, Bornu, Kanem, Borku, Ennedi, London, C. Hurst \& Compagny.

\section{SALI BABANI}

1998 «Ahmadou Bouhari, Lamido de Mindif (1955-1991)», in T. M. BAH (dir.), Acteurs de l'histoire au Nord-Cameroun, XIX et $\mathrm{XX}^{e}$ siècles, Ngaoundéré Anthropos, vol. III, numéro spécial $1: 227-254$.

STILWELL, S.

2000 «Power, Honour and Shame: The Ideology of Royal Slavery in the Sokoto Caliphate », Africa, 70 (3) : 394-421.

Taguem Fah \& Lamblin, G.

2003 «Crise d'autorité, regain d'influence et pérennité des lamidats peuls du Nord-Cameroun », in C.-H. Perrot \& F.-X. Fauvelle-Aymar (dir.), Le retour des rois : les autorités traditionnelles et l'État en Afrique contemporaine, Paris, Karthala : 265-288.

\section{RÉSUMÉ}

L'esclavage est une pratique ancienne dans la partie septentrionale du Cameroun. Consubstantiel à l'apparat et aux modes de production économique des États précoloniaux, le phénomène connaît des permanences et des mutations. Jusqu'à la colonisation européenne, les razzias permettaient aux détenteurs du pouvoir de collecter du bétail humain pour la vente, les travaux champêtres, les harems ou les travaux domestiques. Si le colonisateur abolit la traite humaine, il s'accommoda cependant d'une forme édulcorée d'esclavage royal, sans pour autant améliorer le statut social de l'esclave dans les zones rurales. Le stigmate servile y persiste, les liens de dépendance et d'exploitation y perdurent et suscitent des drames individuels et familiaux. Cependant, I'instruction, l'enrichissement et l'ouverture démocratique sont des facteurs de changement; en dépit de l'illégitimité et de l'illégalité de leur condition, les esclaves apprennent à s'adapter au stigmate servile qu'ils traînent tandis que les maîtres ont profil bas et offrent ou accèdent bien volontiers aux demandes d'affranchissement. L'article comporte des témoignages de personnes d'ascendance servile.

\section{ABSTRACT}

Slaves Bear Testimony in Northern Cameroon. - Slavery, an ancient practice in northern Cameroon, is consubstantial with the pomp and economic modes of production of precolonial states. Till the era of European colonization, raiding enabled rulers to obtain human livestock for sale or for labor in the fields, harems or household chores. Although they abolished the slave trade, colonial authorities put up with a watered-down form of royal slavery and did not improve slaves' social status 
in rural areas. The stigmata of slavery persist; ties of dependency and exploitation survive, still causing dramatic events for families and individuals. Education, growing wealth and increasing democracy are working in favor of change however. Despite the illegitimacy and illegality of their condition, slaves are learning to adapt. They still bear the stigma; but masters, adopting a low profile, willingly propose or accept manumission. Testimonies from persons whose foreparents were slaves.

Mots-clés/Keywords : Nord-Cameroun, affranchissement, esclavage, exploitation, refoulement, servitude/Nothern Cameroon, slavery, exploitation, suppressed feelings, manumission, repression. 